He has been well or even slightly hypomanic since. Ultrasound scan of his kidneys showed a number of small cysts in the right kidney and a single large cyst in the left kidney, but his renal function is normal. He has a younger brother who is well psychiatrically.

Unfortunately, there are insufficient surviving members of Mrs F's family to allow gene linkage studies and there was a resistance within the family for further ultrasound scanning.

ADPKD is the most common genetic disease (Gabow, 1990). The clinical phenotype can result from one of two gene defects. One of these is located on the short arm of chromosome 16 , while some $4 \%$ of families with the disorder have an unknown mutation elsewhere in the genome (Parfrey et al, 1990). There is a suggestion that the q21-22 region of chromosome 11 may be a promising area to examine for genes predisposing to major mental illness (St Clair et al, 1990) and particularly that bipolar affective disorders may be linked to DNA markers on chromosome 11 (Egeland et al, 1987).

Despite the difficulty in completing investigations of our patients it is a rare combination of circumstances and we felt that the case should be reported so that if any other clinicians have a similar family it may be possible to take the issue further.

Egeland, J., Gerhard, D. S., Pauls, D. E., et al (1987) Bipolar affective disorders linked to DNA markers on chromosome 11. Nature, 325, 783-787.

GABOW, P. A. (1990) Autosomal dominant polycystic kidney disease - more than a renal disease. American Journal of Kidney Diseases, 16, 403-413.

Parfrey, P. S., Bear, J. C., Morgan, J., et al (1990) The diagnosis and progress of autosomal dominant polycystic kidney disease. New England Journal of Medicine, 323, 1085-1090.

St Clair, D., BlaCkwood, D., Mutr, W., et al (1990) Association within a family of balanced autosomal translocation with major mental illness. Lancet, 336, $13-16$.

K. WYLIE

D. DE Silva

T. JERRAM

University of Leeds

R. H.S. MindHAM

High Royds Hospital

Menston

Ilkley

West Yorkshire LS296AQ

Down's syndrome, longevity, and Alzheimer's disease

SIR: Prasher (Journal, November 1992, 161, 722) comments on the rare elderly Down's syndrome individuals who have not developed Alzheimer's disease, either clinically or neuropathologically. Dr Prasher is right to suggest that these cases are of interest, since they may also shed light on the role of the $\beta$-amyloid precursor protein (APP) gene in both disorders.

There is now strong evidence that abnormalities in the APP gene and its products are a central mol- ecular event in the aetiology and pathogenesis of Alzheimer's disease. The APP gene is located on chromosome 21. In Alzheimer's disease associated with Down's syndrome, the cause is thought to be overexpression of the APP gene concomitant with the extra copy of genes on chromosome 21 . It is therefore of significance that a small number of Down's syndrome cases are not due to complete trisomy of chromosome 21 , but merely part of it; crucially, this 'obligatory' Down's syndrome region does not include the APP gene locus (Korenberg et al, 1990). Thus, the Down's syndrome individuals who die without Alzheimer-type pathology may be those without triplication of the APP gene. This could be tested by a combination of cytogenetics and subsequent neuropathological analysis.

Korengerg, J. R., Kawashima, H., Pulst, S.-M., et al (1990) Molecular definition of a region of chromosome 21 that causes features of the Down syndrome phenotype. American Journal of Human Genetics, 47, 236-246.

PaUl Harrison

University Department of Psychiatry

Warneford Hospital

Oxford $O X 37 J X$

\section{Eating disorders in Hong Kong}

SIR: Lee et al's report on bulimia nervosa in Hong Kong (Journal, October 1992, 161, 545-551) and their earlier report on anorexia nervosa in Hong Kong (1989), raise the interesting question of the extent of sociocultural factors in the aetiology of anorexia nervosa. This disorder, once seen primarily among the affluent white populations, has now been widely reported in developing countries and Eastern cultures.

Our study in West Malaysia, a multiracial developing country, supports Lee's studies that eating disorders are rare. We examined the ward registers of admissions to male and female psychiatric wards in the University of Malaya from 1970 to 1988 . No male cases were detected in approximately 8000 male psychiatric admissions. Out of over 9000 female admissions, 15 cases fulfilled two or three of the criteria for anorexia nervosa (weight loss, amenorrhoea, and characteristic psychopathology). There were 13 Chinese, one Malay and one Indian. One had a history of bingeing. There was only one case of bulimia nervosa. She was a 24-year-old girl who had a four-year history of binge eating. Lee pointed out that body-image disturbance has not been found among anorexia nervosa subjects in India and that the diagnostic criteria may need to be modified in 